JPE 12-5-16

http://dx.doi.org/10.6113/JPE.2012.12.5.830

\title{
A Fuzzy Based Solution for Allocation and Sizing of Multiple Active Power Filters
}

\author{
Amir Moradifar ${ }^{\dagger}$ and Hassan Rezai Soleymanpour* \\ ${ }^{\dagger *}$ Dept. of Electrical and Computers Eng., The University of the Semnan, Semnan, Iran
}

\begin{abstract}
Active power filters (APF) can be employed for harmonic compensation in power systems. In this paper, a fuzzy based method is proposed for identification of probable APF nodes of a radial distribution system. The modified adaptive particle swarm optimization (MAPSO) technique is used for final selection of the APFs size. A combination of Fuzzy-MAPSO method is implemented to determine the optimal allocation and size of APFs. New fuzzy membership functions are formulated where the harmonic current membership is an exponential function of the nodal injecting harmonic current. Harmonic voltage membership has been formulated as a function of the node harmonic voltage. The product operator shows better performance than the AND operator because all harmonics are considered in computing membership function. For evaluating the proposed method, it has been applied to the 5-bus and 18-bus test systems, respectively, which the results appear satisfactorily. The proposed membership functions are new at the APF placement problem so that weighting factors can be changed proportional to objective function.
\end{abstract}

Key words: Active Power Filter placement, Fuzzy set theory, Particle Swarm Optimization

\section{INTRODUCTION}

With increasing use of power electronic equipment and nonlinear loads, the level of voltage harmonic distortion in distribution networks is significantly increasing. Nonlinear loads act as current sources injecting harmonic currents into the power systems. These power-electronic-based loads have caused severely distorted voltage waveforms at the point of common coupling (PCC). As harmonics propagate through the system, they result in increased losses and possible equipment loss-of-life. Also over currents or over voltages resulting from resonances can damage equipment. Additionally, harmonics can interfere with control, communication, and protective equipment [1]. With this reasons different research institutes have studied about the proper limitation of harmonic disturbance levels and released different standards. The conventional solutions include network reconfiguration, capacitor switching and passive filtering. Widespread increase in number of small power electronics based apparatus causes a higher level of harmonic distortions in power systems. In this

Manuscript received Jan. 4, 2012; revised Jul. 30, 2012

Recommended for publication by Associate Editor Kyo-Beum Lee.

${ }^{\dagger}$ Corresponding Author:Moradifar.Amir@gmail.com

Tel: +98-231-33-54264, Fax: +98-231-33-54123, Semnan University

* Dept. of Electrical and Computers Eng., The University of the Semnan, Iran case, the above-mentioned solutions for large sources of harmonics may become inappropriate or ineffective. Therefore, the need for a better and effective solution, such as active power filter (APF) which is also called active power line conditioner (APLC) [2] is essential, which could control the harmonic levels in the presence of wide-variation of harmonic sources and system impedances [3]. APFs provide injected equal-but-opposite currents to the PCC that completely eliminate the nonsinusoidal requirements of the nonlinear loads.

The installation of an APF in an appropriate place and proper size was developed in research from 1990 to present [4]-[16]. The important factors to be considered for APF applications are: existing harmonic pollution levels, harmonic standard constraints, locations and sizes of APFs and finally network topology. The size of an APF is normally defined as its maximum effective injection current. As the essential part of the objective function, total harmonic distortion of voltage (THDv) level and APFs size have been considered in [4], [5]. Harmonic transmission-line loss (HTLL) and motor load loss (MLL), due to harmonics and telephone influence factor (TIF), are included in the objective function in other studies [6], [9].

Analytical [9], [10], mathematical programming [11] and a host of other methods have been developed to solve the problem. The optimization problem, however, still remains tempting enough for the application of the new solution tools. 
Although the analytical optimization algorithms do not suffer from a time-consuming problem, the local minimum is their main drawback. The need for the initial solution and difficulty in differentiation from various types of nonlinear objective functions is among other disadvantages of these methods. It should be noticed that an incorrect selection of initial values leads to inaccurate results when these methods are applied.

Artificial intelligence (AI) techniques have been tried in recent years in search of a superior solution tool. Among the AI techniques, evolutionary computing methods such as genetic algorithm (GA) [12], particle swarm optimization (PSO) [13], bacterial foraging technique [14], ant colony optimization [15] and tabu search [16] have been reported to produce superior results. However, one common drawback of these techniques lies in the huge computing task involved in obtaining the solution. Another AI approach involves fuzzy logic. Fuzzy based approaches involve less computational burden. No fuzzy approach so far has been proposed the APF placement problem. Many fuzzy based methods have been reported about the capacitor placement problem [17]-[27]. These methods, however, do not always find the best possible solutions, but they generally can find an acceptable sub-optimal solution using less computing cost.

Fuzzy based solution methods need modeling of the system through fuzzy membership functions. Identification of proper membership function is the most challenging task in the development of fuzzy based solution techniques. Whatever may be the objective of placing APFs in distribution system, they basically inject harmonic current to the network as a result of which the system harmonic voltage and THDv improve and bus injecting harmonic currents decrease. Thus, bus harmonic voltages measures and bus injecting harmonic currents in the network have been utilized as indicators for deciding the location and also determining the size of the APFs.

In this paper, a combination of Fuzzy-MAPSO method is implemented to determine the optimal allocation and size of APFs. A fuzzy based method is used which basically identifies probable APF location using fuzzy approach. The modified adaptive particle swarm optimization (MAPSO) technique is used for final selection of the APF size. Simulation results are finally compared with the results obtained by another heuristic method (GA in this case) and also an analytical optimization technique to validate the capability of the proposed method.

\section{PROBLEM FORMULATION}

In this paper, the aim is to minimize injected currents of APFs as the objective function while harmonic standard levels and APF current constraints are simultaneously observed. The size of APFs can be considered either continuous or discrete.

\section{A. APF Model}

The selected model for the APF in this paper is a current source injecting harmonic currents into the power system. These currents consist of two components, real and imaginary parts. The indices $r$ and i represent the real and imaginary parts of an APF current, respectively, as:

$$
I_{F, m}^{h}=I_{F, m}^{h, r}+I_{F, m}^{h, i}
$$

where, $I_{F, m}^{h}$ denote the APF current at bus $m$ for harmonic order h. $I_{F, m}^{h, r}$ and $I_{F, m}^{h, i}$ denote the real and imaginary part of APF current at bus $m$ for harmonic order $h$, respectively.

The Root Mean Square (RMS) current of each APF is determined as follows:

$$
I_{F, m}=\left[\sum_{h=2}^{H}\left(I_{F, m}^{2}{ }^{h, r}+I_{F, m}^{2}{ }^{h, i}\right)\right]^{1 / 2}
$$

\section{B. Objective Function}

From The most important objectives of placement and sizing of APFs in a power system are to minimize the cost of APFs. The constraints also maintain size of APFs, individual and total harmonic distortions within a standard level. The size of APFs can be considered either continuous or discrete. It is undeniable that the case with no current constraints for APFs is impractical because the DC source in the APF is implemented by capacitors and inductors, which are discrete devices. The resulting optimization problem can be presented as follows:

$$
\operatorname{Min} \sum_{m=1}^{M} I_{F, m}
$$

Such that:

$$
\begin{gathered}
I_{F, m} \leq \bar{I}_{F, M A X}, \quad m=1,2, \ldots M \\
I_{F, m} \in D \\
\left|V_{k}^{h}\right| \leq \bar{V}_{k}^{h}, \quad h=2, \ldots, H, \quad k=1, \ldots, K \\
T H D v_{k} \leq \overline{T H D} v_{k} \quad k=1, \ldots, K
\end{gathered}
$$

where, $\mathrm{H}$ and $\mathrm{K}$ denote the maximum considered harmonic order and number of buses, respectively. $I_{F, m}$ and $\bar{I}_{F, M A X}$ denote the size of APF in the candidate bus $\mathrm{m}$ and APF maximum current, respectively. $\left|V_{k}^{h}\right|$ and $\bar{V}_{k}^{h}$ denote the amplitude of harmonic voltage $h$ in bus $k$ and maximum permissible value for harmonic voltage $h$ in bus $k$, imposed by harmonic standards, respectively. $T H D v_{k}$ and $\overline{T H D} v_{k}$ denote the THDv in bus $k$ and maximum THDv in bus $k$, imposed by harmonic standards, respectively.

$I_{F, m}$ is the size of the APF in the candidate bus $\mathrm{m}$, its value 
is equal to the smallest value of set $\mathrm{D}$ which is greater than $I_{F, m}$. If APF sizes are considered discrete, D is a set of discrete values including zero and permissible values. If APF sizes are considered continuous, then $\mathrm{D}$ includes a set of real and non-negative values.

$V_{k, \text { new }}^{h}$ is the voltage phasor for harmonic $\mathrm{h}$ in bus $\mathrm{k}$ after APF is installed. It can be determined as follows:

$$
V_{k, \text { new }}^{h}=V_{k, \text { old }}^{h}+\Delta V_{k}^{h}
$$

where $V_{k, \text { old }}^{h}$ is the voltage phasor for harmonic $\mathrm{h}$ in bus $\mathrm{k}$ before APF installation and $\Delta V_{k}^{h}$ is determined as

$$
\Delta V_{k}^{h}=\sum_{m=1}^{M} Z_{k, m}^{h} I_{m}^{h}
$$

It can be seen that $V_{k \text {,new }}^{h}$ is a function of bus $\mathrm{k}$ voltage before APF installation, network impedance matrix for harmonic $\mathrm{h}$ and injected APF current for this harmonic. In the optimization problem, $V_{k, \text { old }}^{h}$ and $\mathrm{Z}$ for each harmonic are considered known and injected currents of APFs are considered as decision-making variables. Using (10) and (11), the real and imaginary parts of $V_{k, \text { new }}^{h}$ can be determined as follows [12]:

$$
\begin{aligned}
& V_{k, \text { new }}^{h, r}=V_{k, \text { old }}^{h, r}+\sum_{m=1}^{M}\left(Z_{k, m}^{h, r} I_{m}^{h, r}-Z_{k, m}^{h, i} I_{m}^{h, i}\right) \\
& V_{k, \text { new }}^{h, i}=V_{k, \text { old }}^{h, i}+\sum_{m=1}^{M}\left(Z_{k, m}^{h, r} I_{m}^{h, i}+Z_{k, m}^{h, i} I_{m}^{h, r}\right) \\
& V_{k, \text { new }}^{h}=V_{k, \text { new }}^{h, r}+j V_{k, \text { ew }}^{h, i}
\end{aligned}
$$

By determining $V_{k, \text { new }}^{h}$, the dependent function $T H D v_{k}$ can be calculated as

$$
T H D v_{k}=\sqrt{\sum_{h \geq 2}^{H}\left(\frac{\left|V_{k, n e w}^{h}\right|}{\left|V_{k}^{1}\right|}\right)^{2}}
$$

We have nonlinear loads current ( $\left.I_{\text {nld }}\right)$ and bus admittance matrix for harmonic order $\mathrm{h}$ is also presented by $Y_{\text {bus }}^{h}$ therefore the bus voltages can be attained as

$$
V_{\text {bus }}^{h}=\left[Y_{\text {bus }}^{h}\right] \bullet I_{l}^{h}
$$

where $V_{\text {bus }}^{h}$ and $I_{l}^{h}$ denote the bus harmonic voltages and injecting harmonic current vectors for harmonic order $h$, respectively. Considering an APF located at each bus, the bus injecting current is implied as

$$
I_{l}^{h}=I_{f}^{h}-I_{n l d}^{h}
$$

where $I_{f}^{h}$ and $I_{n l d}^{h}$ denote the APF and nonlinear load current vectors for harmonic order h, respectively. Therefore we can determine the injecting harmonic current $\left(I_{l}^{h}\right)$ and it dependent function, the total harmonic distortion of current (THDi) can be calculated as

$$
T H D i_{\text {line }}=\sqrt{\sum_{h \geq 2}^{H}\left(\frac{\left|I_{\text {line }}^{h}\right|}{\left|I_{\text {line }}^{1}\right|}\right)^{2}} \quad \text { line }=1,2 \ldots, L
$$

where $\mathrm{L}$ is the number of lines.

\section{FUZZY SET THEORY FUNDAMENTALS}

The fuzzy set, which is a generalization of the conventional crisp set, extends the values of set membership from values in $\{0,1\}$ to the unit interval $[0,1]$. A fuzzy set can be defined mathematically by assigning a value to each possible element of membership in the set. Some basic fuzzy operators are introduced in this section.

The AND operation (intersection of membership functions): Let $A$ and $B$ be two fuzzy sets with membership functions $\mu_{A}(x)$ and $\mu_{B}(x)$, respectively, and $X$ be the universal set. The membership function of the intersection (AND) is defined as

$$
\mu_{A \cap B}(x)=\min \left(\mu_{A}(x), \mu_{B}(x)\right) \quad x \in X .
$$

The OR operation (union of membership functions): The membership function of the union (OR) is defined as

$$
\mu_{A \cup B}(x)=\max \left(\mu_{A}(x), \mu_{B}(x)\right) \quad x \in X .
$$

Product of two fuzzy sets: The product of two fuzzy sets has a membership function defined by

$$
\mu_{A . B}(x)=\mu_{A}(x) * \mu_{B}(x) \quad x \in X .
$$

A complete review on fuzzy set theory fundamentals and fuzzy decision making can be found in [28]. 


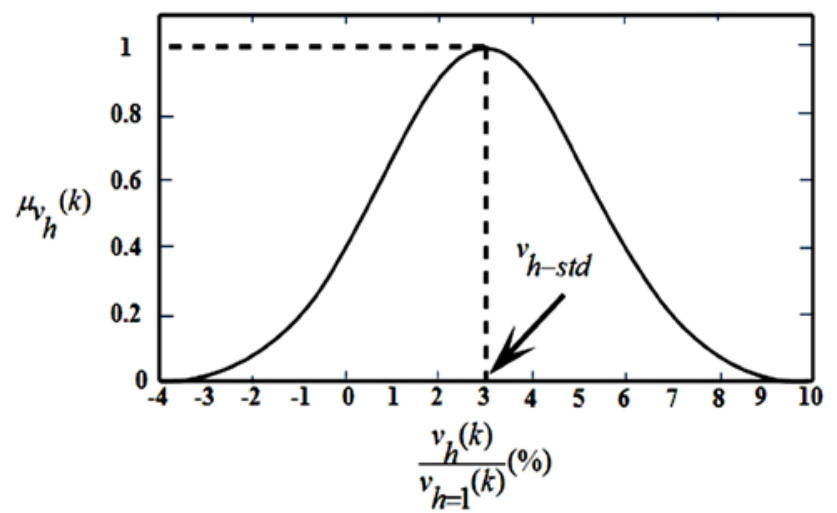

Fig. 1. Proposed membership function for the sensitivity of the harmonic voltage without modification.

\section{FUZZY MODELING}

We define two membership function is the following. The first membership function $\mu_{v_{h}}(k)$ for the harmonic voltages of bus $\mathrm{k}$ is defined as (23) and is shown in Fig. 1.

$\mu_{v_{h}}(k)=\exp \left(-w_{v}\left(\frac{\left(\frac{v_{h}(k)}{v_{h=1}(k)}\right)-v_{h-s t d}}{v_{h-s t d}}\right)^{2}\right)$

where $w_{v}$ denote the weighting factor for harmonic voltage. $v_{h}(k)$ and $v_{h-s t d}$ denote the amplitude and maximum permissible value imposed by standards for harmonic voltage order h in bus $\mathrm{k}$, respectively.

We modify the membership function of the harmonic voltages as (21) and Fig. 2. In this membership function, a bus with the higher harmonic voltage than $v_{h-s t d}$ is given a low value, whereas a bus with the lower harmonic voltage than $v_{h-s t d}$ is set to one. The membership function is defined as

$$
\begin{aligned}
& \mu_{v_{h}}(k)= \\
& \left\{\begin{array}{l}
\exp \left(-w_{v}\right. \\
1
\end{array}\right.
\end{aligned}
$$$$
\left\{\exp \left(-w_{v}\left(\frac{\left(\frac{v_{h}(k)}{v_{h=1}(k)}\right)-v_{h-s t d}}{v_{h-s t d}}\right)^{2}\right) \quad \text { if } \frac{v_{h}(k)}{v_{h=1}(k)} \geq v_{h-s t d}\right.
$$

otherwise

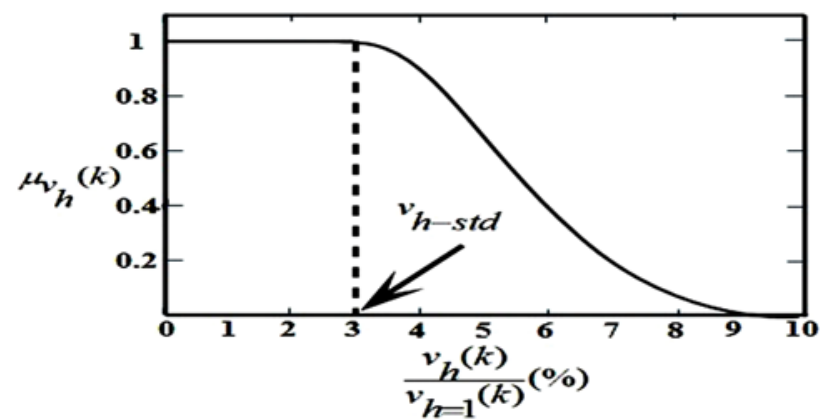

Fig. 2. Proposed membership function for the sensitivity of the harmonic voltage with modification.

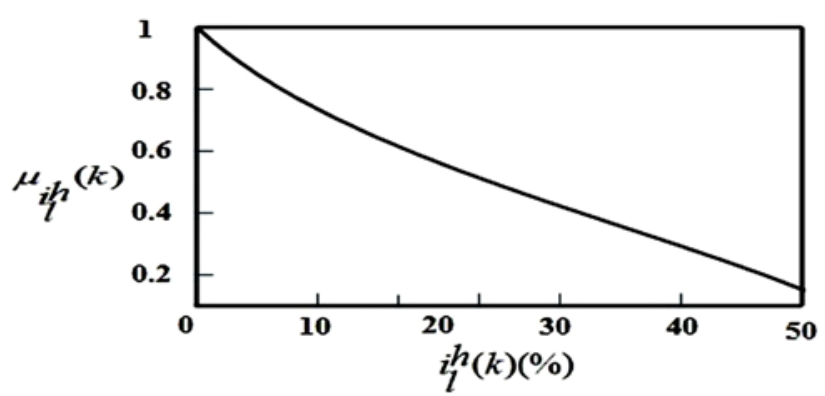

Fig. 3. Proposed membership function for the sensitivity of the harmonic current.

The second membership function $\mu_{i_{l}^{h}}(k)$ defined for injecting harmonic current into the $l$ line for bus $\mathrm{k}$ in harmonic order $\mathrm{h}$ is depicted as (22) and Fig. 3. In this membership function, a high harmonic current is given a low value.

$$
\mu_{i_{l}^{h}}(k)=\exp \left(-w_{i}\left(\frac{i_{l}^{h}(k)}{T H D_{i-\text { total }}}\right)\right)
$$

where $w_{i}$ and $T H D_{i-t o t a l}$ denote the weighting factor for harmonic current and summation of THDi in the lines, respectively.

We use AND operator and product operator for identification of probable APF locations in distribution systems. Product operator for sharing all harmonics to compute membership function shows better performance than AND operator. For this reason, we use it for harmonic voltage as follows:

$$
\mu_{v}(k)=\text { product }\left\{\mu_{v_{h}}(k)\right\}
$$

We use product operation for harmonic currents at (24) and (25) too. 


$$
\begin{aligned}
& \mu_{i_{l}}(k)=\text { product }\left\{\mu_{i_{l}^{h}}(k)\right\} \\
& \mu_{i}(k)=\text { product }\left\{\mu_{i_{l}}(k)\right\}
\end{aligned}
$$

Subsequently, apply the Product operator on the membership functions of $\mu_{v}(k)$ and $\mu_{i}(k)$ to determine an optimum bus for the installation of the APF. Here, the optimum bus is the one with the lowest membership function evaluated the following expression:

$$
\mu_{s}(k)=\operatorname{product}\left\{\mu_{v}(k), \mu_{i}(k)\right\}, \quad k=1,2, \ldots, K .
$$

and

$$
\mu_{s}\left(k^{*}\right)=\min \left\{\mu_{s}(1), \mu_{s}(2), \ldots, \mu_{s}(K)\right\}, k=1,2, \ldots, K
$$

where $k^{*}$ is the bus with the lowest membership function and is the optimum bus selected for installation of the APF. The optimum size of APF selected for the installation is the quantity which has the lowest cost (injecting current) while satisfying the size of APFs, individual and total harmonic distortion constrains.

\section{OVER VIEW OF MAPSO}

Particle swarm optimization is firstly introduced in [29] as a modern heuristic optimization technique. PSO is a population based search algorithm dealing with a swarm of random solutions (so called particles) in search space. Particles adjust their positions in search space by using of velocity vectors defined for the particles and depend on the best experiences they have found until now. In each iteration of search process the position and velocity of each particle is updated based on two following formula:

$$
\begin{aligned}
V_{i, \text { iter }+1}= & w V_{i, i t e r}+c_{1} r_{1}\left(P_{i, i t e r}^{\text {best }}-X_{i, i t e r}\right) \\
& +c_{2} r_{2}\left(G_{i t e r}^{b e s t}-X_{i, i t e r}\right) \\
X_{i, \text { iter }+1}= & X_{i, \text { iter }}+V_{i, \text { iter }+1}
\end{aligned}
$$

where $V_{i, i t e r}$ and $X_{i, i t e r}$ represent the velocity vector and the position vector of ith particle at iteration iter, respectively; $P_{i, i t e r}^{\text {best }}$ and $G_{i t e r}^{\text {best }}$ are personal best position (experience) of ith particle and global best position of swarm until iteration iter, respectively; w is called inertia weight factor, which controls the global and local exploration capabilities of the particle; $c_{1}$ and $c_{2}$ are cognitive and social coefficients, respectively, which balance the acceleration of the particle toward $P_{i, \text { iter }}^{\text {best }}$ and $G_{i t e r}^{\text {best }} ; \mathrm{r}_{1}$ and $\mathrm{r}_{2}$ are two random numbers between 0 and 1 used for increasing the stochastic behavior of the algorithm. There is also an upper limit, velocity limiter, for the velocities of particles in all dimensions to prevent the particles from moving too rapidly from one region in search space to another. This velocity limiter is usually considered as a proportion of the allowable position range.

Though the PSO can solve many optimization problems, it is weak dealing with complex problems like the problem in our case. Several modified versions of PSO have been invented by the scholars in the field of optimization to decrease the weaknesses of the PSO. MAPSO is one of those versions which was introduced in [30] and was tightly tested on several cases of a complex practical problem.

There are three basic modifications in the MAPSO, which make the algorithm to become more powerful against complex problems. These modifications are: 1) splitting up the cognitive part into Best and Not-Best components, which helps particles to search the solution space more efficiently compared with the PSO, 2) using the personal best position exchanging, which causes more activity in particles, and 3) implementing of an adaptive velocity limiter with which the MAPSO can benefit from both good exploration capability and convergence behavior by linearly decreasing the permission of particles jumping. As well as implementing these modifications, MAPSO also benefits from the concepts of time varying inertia weight (TVIW), time varying acceleration coefficients (TVAC) and a special type of the tree topology. These complementary modifications help the MAPSO to become strong versus complicated optimization problems [30].

In this paper, MAPSO technique has been used for final selection of the APF size with meeting IEEE-519 constrains and size of APF.

The population of particles $X_{i}$ (consisting of real and imaginary parts of APF current at all buses for all harmonic orders) as well as their velocity $V_{i}$ in the search space are initialized. Therefore, $X$ vector can be considered as a set of $I_{F, m}^{h, r}$ and $I_{F, m}^{h, i}$ and $V$ vector as set of $V_{F, m}^{h, r}$ and $V_{F, m}^{h, i}$. If $\mathrm{H}$ represents the number of harmonic under consideration and $\mathrm{M}$ as the number of candidate buses, each row of vectors consists of $2 \mathrm{HM}$ particles. Vectors $X$ and $V$ are described as shown in (30) and (31).

$$
\begin{array}{r}
X_{j}=\left[I_{F, 1}^{1, r}, I_{F, 2}^{1, r}, \ldots, I_{F, M}^{1, r}, I_{F, 1}^{2, r}, I_{F, 2}^{2, r}, \ldots I_{F, M}^{2, r}, \ldots\right. \\
, I_{F, 1}^{H, r}, I_{F, 2}^{H, r}, \ldots, I_{F, M}^{H, r}, I_{F, 1}^{1, i}, I_{F, 2}^{1, i}, \ldots, I_{F, M}^{1, i}, I_{F, 1}^{2, i}, \\
\left.I_{F, 2}^{2, i}, \ldots I_{F, M}^{2, i}, \ldots, I_{F, 1}^{H, i}, I_{F, 2}^{H, i}, \ldots, I_{F, M}^{H, i}\right] \\
V_{j}=\left[V_{F, 1}^{1, r}, V_{F, 2}^{1, r}, \ldots, V_{F, M}^{1, r}, V_{F, 1}^{2, r}, V_{F, 2}^{2, r}, \ldots V_{F, M}^{2, r}, \ldots\right. \\
, V_{F, 1}^{H, r}, V_{F, 2}^{H, r}, \ldots, V_{F, M}^{H, r}, V_{F, 1}^{1, i}, V_{F, 2}^{1, i}, \ldots, V_{F, M}^{1, i}, V_{F, 1}^{2, i}, \\
\left.V_{F, 2}^{2, i}, \ldots V_{F, M}^{2, i}, V_{F, 1}^{H, i}, V_{F, 2}^{H, i}, \ldots, V_{F . M}^{H, i}\right]
\end{array}
$$




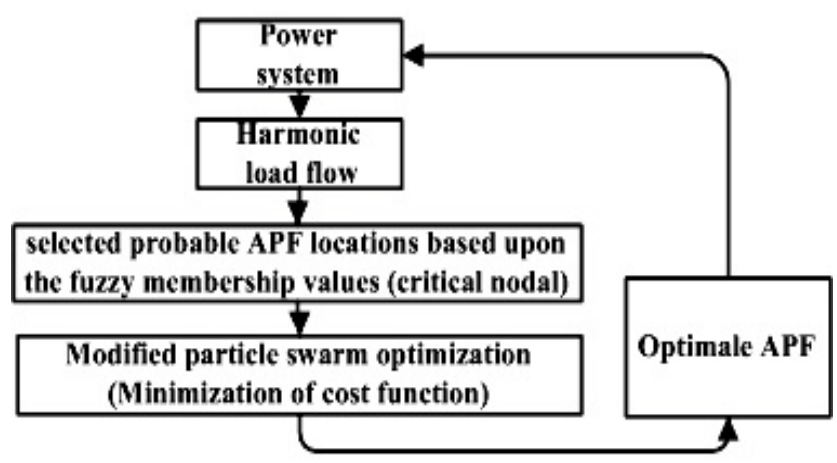

Fig. 4. Bloc of intelligent fuzzy-MAPSO approach.

\section{IMPLEMENTATION OF FUZZY-MAPSO FOR APFs ALLOCATION AND SIZING PROBLEM}

The solution algorithm consists of two stages. The first stage selects a number of probable APF locations and also allocates some APFs at the selected locations based upon the fuzzy membership values. While allocating the APFs at the selected locations, we however increment the smallest APF unit at a time. This is done to have a better selection of the candidate node as the addition of an APF unit may change the relative memberships of the nodes.

In the second stage of the solution algorithm, we decide the APF sizes at the selected locations using the MAPSO. The APF sizes found in the previous stage are used as the starting APF sizes. The principle of method is presented in Fig. 4.

The complete solution algorithm is as given below.

Step 1: In this step, the power system configuration and data, nonlinear loads location, current ( $\left.I_{n l d}\right)$, and constraints, such as maximum-allowed individual harmonic distortion, THDv, number and size of APFs are specified. The number of population members, the number of iterations and other parameters of MAPSO are also set in this step.

Step 2: Evaluate the membership functions $\mu_{v}$ and $\mu_{i}$ for the nodes using (23) and (25), respectively.

Step 3: Identify the candidate node using fuzzy decision rule, using (26) and (27).

Step 4: Add minimum APF unit at the selected node.

Step 5: Perform harmonic load flow with the added APF.

Step 6: If APF addition violates maximum node harmonic voltage limit, remove the location for further consideration and register this attempt as 'failed'.

Step 7: Repeat from step 2, until the desired numbers of candidate APF nodes are selected or sufficient numbers of failed attempts have been registered.

Step 8: Apply MAPSO to determine optimum APF sizes.

The proposed method was applied to the test systems shown in Fig. 5.

By following implicated steps for a radial distribution networks, the objective value (optimal site and size of APFs) can be found for specific weighting factors of harmonic voltage

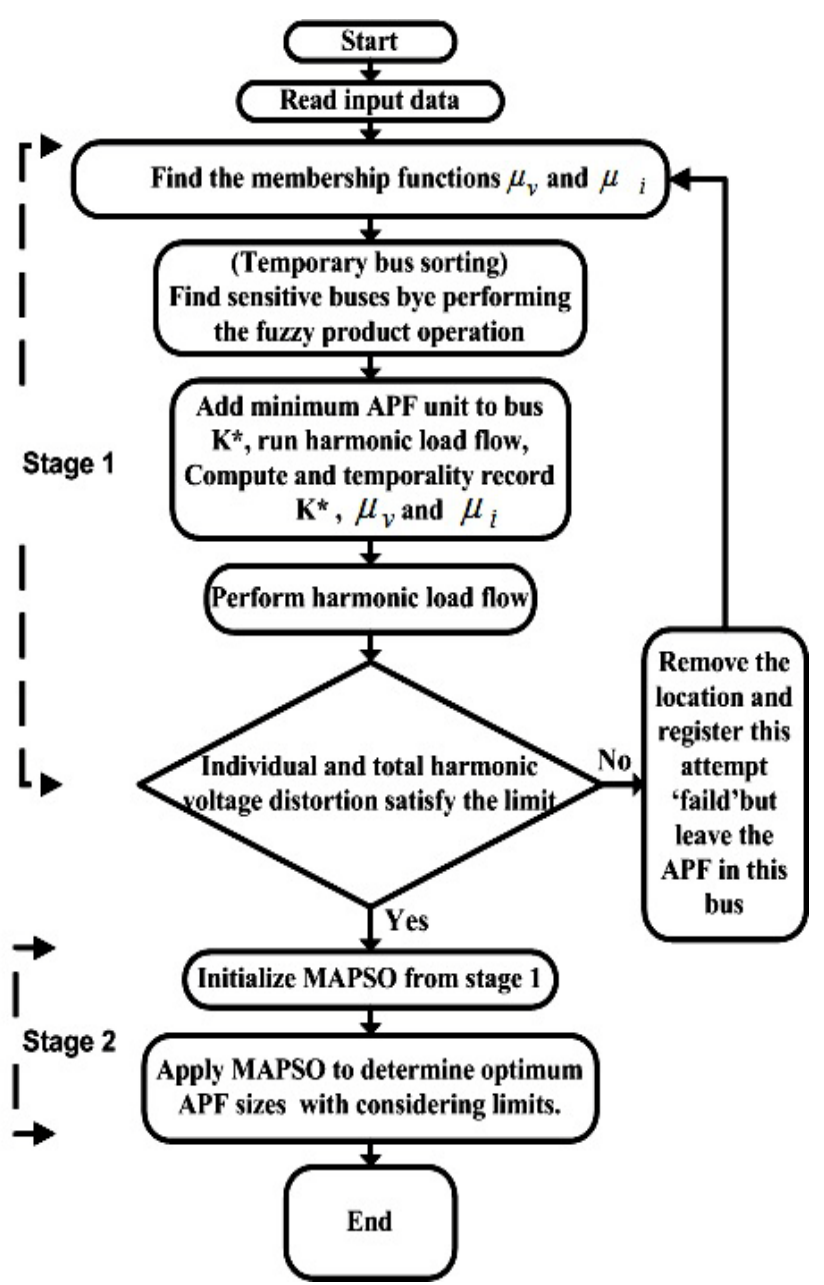

Fig. 5. Flowchart of the proposed method.

and current membership functions ( $w_{v}$ and $w_{i}$ ).

We consider the weighting factors for harmonic voltage membership ( $w_{v}$ ) and harmonic current membership ( $\left.w_{i}\right), 0.6$ and 0.4 , respectively, because in our objective function, the distortions of voltage more important than the distortions of current. The weighting factors can be selected properly in case the other objective functions such as TIF, MLL and HTLL are considered. For example, the harmonic currents are more important than the harmonic voltages in TIF objective function. On the other hand, it is clear that the amount of the membership functions had a clear effect on the objective values. Therefore finding the best values for $w_{v}$ and $w_{i}$ is our great goal for providing the optimum amount for the objective function in the next research, so steps 1 thorough 8 can be placed in a loop of other optimization algorithm. Decision variables of the optimization algorithm will be defined as two weighting factors which are $w_{v}$ and $w_{i}$. The whole process, step 1 through 8 , will be run for different values of the parameters ( $w_{v}$ and $w_{i}$ ), so the hybrid method will find the best APF plan. 
TABLE I

APF CURRENT RATINGS

\begin{tabular}{|c|c|c|c|c|}
\hline Name of APF & A & B & C & D \\
\hline Rating (\%) & 5 & 4 & 3 & 2 \\
\hline
\end{tabular}

The coding is written in MATLAB 7.8 programming language and executed by using a Pentium Dual core, $3.2 \mathrm{GHz}$, with 4-GB random-access memory (RAM) processor.

\section{SiMULATION RESULtS}

For evaluating the proposed method, it has been applied to the 5-bus and 18-bus test systems, respectively, which the results appear satisfactorily.

The proposed of these cases study are to investigate ability of multiple APFs to minimize cost and to control voltage harmonic distortion to meet IEEE-519 constrains at all buses. Characteristic harmonics through 25th multiple are considered. We assume that four APFs are available, with the current ratings (on $12.5 \mathrm{KV}, 10 \mathrm{MVA}$ system base) given in Table I.

The parameters of the MAPSO have been set as follows based on trial and error (the selected values are the best ones among several runs): $w_{\min }=0.4, w_{\max }=0.9$, $c_{1 b}^{\text {initial }}=1.4, \quad c_{1 b}^{\text {final }}=0.9, \quad c_{1 n b}^{\text {initial }}=0.1, \quad c_{1 n b}^{\text {final }}=0.07$, $c_{2}^{\text {initial }}=0.7, \quad C_{2}^{\text {final }}=1.5, \quad R^{\text {initial }}=0.2, \quad R^{\text {final }}=0.08$, iter $_{\text {max }}=6000$ and $N_{P B E}=15$, where $N_{P B E}$ indicates number of particles participating in the personal best position exchanging in each iteration [30].

\section{A. 5-bus Test System}

An industrial customer will be served by constructing a three-mile $12.5 \mathrm{kV}$ overhead feeder from a dedicated 138/12.5kV substation transformer. The customer will have $5 \mathrm{MW}$ at power factor 0.85 of conventional load, six-pulse adjustable-speed drive (ASD) where the 5th, 7th, 11th, 13th, 17th, 19th, 23rd, and 25th harmonics are produced and injecting $5.17 \%$ harmonic current (RMS). The ASD is connected through a delta-delta transformer (i.e., no phase shift). The customer also has 1800kVAr of shunt power
TABLE II

Bus Data For The 5-Bus System

\begin{tabular}{|c|c|c|c|c|c|c|}
\hline $\begin{array}{c}\text { Bus } \\
\text { num }\end{array}$ & $\begin{array}{c}\text { Bus } \\
\text { type* }\end{array}$ & $\begin{array}{c}\text { P\&Q } \\
\text { Gen. } \\
(\%)\end{array}$ & $\begin{array}{c}\text { P } \\
\text { load } \\
(\%)\end{array}$ & $\begin{array}{c}\text { Q } \\
\text { load } \\
(\%)\end{array}$ & $\begin{array}{c}\text { Desired } \\
\text { voltage } \\
(\%)\end{array}$ & $\begin{array}{c}\text { Shunt } \\
\text { Q } \\
\text { Load(\%) }\end{array}$ \\
\hline 1 & 1 & 0 & 0 & 0 & 102 & 0 \\
\hline 2 & 3 & 0 & 0 & 0 & 0 & 0 \\
\hline 3 & 3 & 0 & 0 & 0 & 0 & 0 \\
\hline 4 & 3 & 0 & 0 & 0 & 0 & -18 \\
\hline 5 & 3 & 0 & 50 & 31 & 0 & 0 \\
\hline
\end{tabular}

*1=Swing Bus, 2=PV Bus, 3=PQ Bus

TABLE III

Line DATA For The 5-Bus System

\begin{tabular}{|l|l|l|l|l|}
\hline $\begin{array}{l}\text { From } \\
\text { Bus }\end{array}$ & $\begin{array}{l}\text { To } \\
\text { Bus }\end{array}$ & $\begin{array}{l}\mathrm{R}(+) \\
(\%)\end{array}$ & $\begin{array}{l}\mathrm{X}(+) \\
(\%)\end{array}$ & $\begin{array}{l}\text { Line } \\
\text { Charging } \\
(\%)\end{array}$ \\
\hline 1 & 2 & 0.04 & 0.25 & 65 \\
\hline 2 & 3 & 0.33 & 7 & 0 \\
\hline 3 & 4 & 4.06 & 11.96 & 0.0314 \\
\hline 4 & 5 & 0.67 & 6.67 & 0 \\
\hline
\end{tabular}

factor correction capacitors. The one-line diagram on a 10 MVA base is shown in Fig.6. Details of the test system data are given in Tables II and III.

Voltage and current distortions for all harmonic orders and at all buses as well as THDv and THDi can be calculated by using the admittance matrix for all harmonic orders and the harmonic contents of nonlinear load with using (8)-(16).

Before applying the proposed method Table IV illustrates the obtained simulation results in no APF state. It can be found from Table IV that the system is badly distorted by nonlinear load. The maximum values of THDv and individual harmonic distortions at all buses are shown as bold in Table VIII. As seen in Table IV, bus 4 has the maximum value of THDv with $7.63 \%$

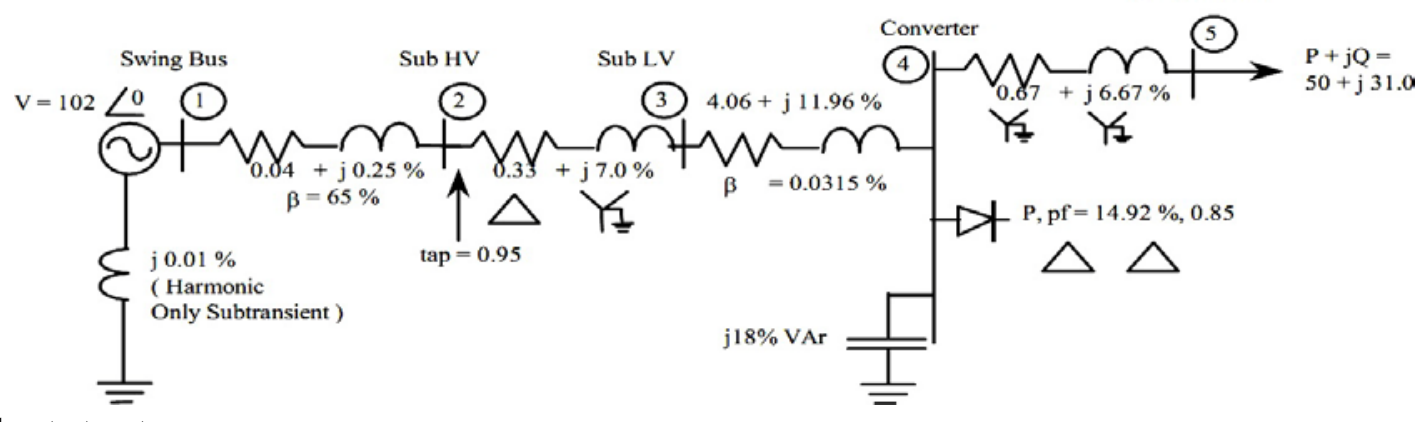

Fig. 6. 5-bus test system. 
TABLE IV

Initial Voltage Distortions For The 5-Bus System

\begin{tabular}{|c|c|c|c|c|c|c|c|}
\hline \multirow{2}{*}{\multicolumn{2}{|c|}{$\begin{array}{c}\text { Harmonic } \\
\text { Order }\end{array}$}} & \multicolumn{5}{|c|}{ Bus number } & \multirow{2}{*}{$\begin{array}{l}\text { AVG } \\
\text { (\%) }\end{array}$} \\
\hline & & 1 & 2 & 3 & 4 & 5 & \\
\hline \multirow{8}{*}{$\begin{array}{l}< \\
0 \\
\stackrel{0}{0} \\
00 \\
0 \\
0 \\
0 \\
0 \\
0 \\
0 \\
0 \\
0 \\
0 \\
0\end{array}$} & 5 & 0 & 0.074 & 1.886 & 5.28 & 4.797 & \\
\hline & 7 & 0 & 0.075 & 1.886 & 5.28 & 4.746 & 2.39 \\
\hline & 11 & 0 & 0.018 & 0.728 & 1.242 & 1.078 & 0.61 \\
\hline & 13 & 0 & 0.012 & 0.285 & 0.795 & 0.681 & 0.35 \\
\hline & 17 & 0 & 0.007 & 0.15 & 0.416 & 0.341 & 0.18 \\
\hline & 19 & 0 & 0.006 & 0.116 & 0.323 & 0.258 & 0.14 \\
\hline & 23 & 0 & 0.004 & 0.077 & 0.211 & 0.161 & 0.09 \\
\hline & 25 & 0 & 0.004 & 0.065 & 0.177 & 0.13 & 0.07 \\
\hline \multicolumn{2}{|c|}{ THDv (\%) } & 0 & 0.11 & 2.79 & 7.63 & 6.89 & 3.48 \\
\hline
\end{tabular}

TABLE V

Harmonic Contents Of The APF At Bus 4

\begin{tabular}{|l|l|}
\hline \multicolumn{1}{|c|}{$\begin{array}{c}\text { Harmonic } \\
\text { order }\end{array}$} & \multicolumn{1}{c|}{$\begin{array}{c}\text { Inject } \\
\text { Current (\%) }\end{array}$} \\
\hline 5 & 1.1397 \\
\hline 7 & 0.9888 \\
\hline 11 & 0.3606 \\
\hline 13 & 0.2074 \\
\hline 17 & 0.1220 \\
\hline 19 & 0.0108 \\
\hline 23 & 0.0051 \\
\hline 25 & 0.0008 \\
\hline RMS & 1.57 \\
\hline
\end{tabular}

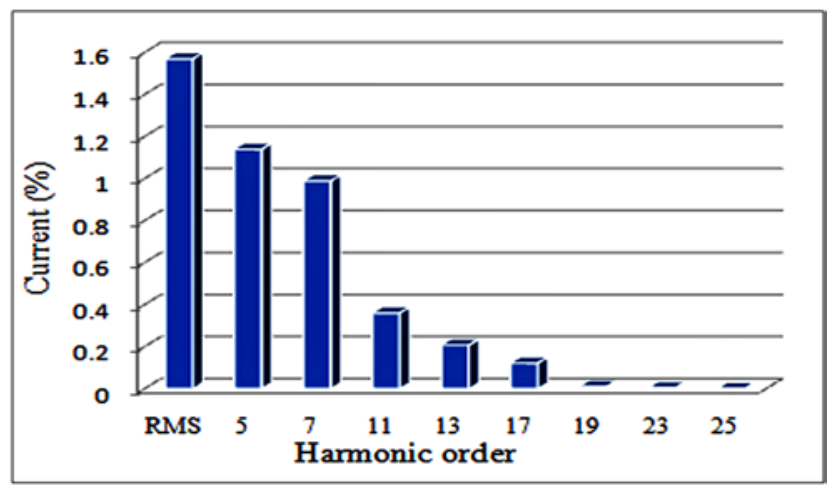

Fig. 7. Harmonic contents of installed APF in bus 4.

After applying the proposed method, D APF is installed on bus 4 . The aim is to meet the harmonic standard levels with minimum injected harmonic currents. The maximum voltage harmonic distortions and THDv are considered to be 3 and 5\%, respectively. The optimum solution results in $1.57 \%$ injection current at bus 4. Injecting harmonic currents and THDv after installation APF, are given in Table V and VI, respectively.

To further clarify the results, Fig. 5 provides the harmonic contents of installed APF in bus 4 .
TABLE VI

Voltage Conditions After Employing The Proposed Method For The 5-Bus System

\begin{tabular}{|c|c|c|c|c|c|c|c|}
\hline \multirow{2}{*}{\multicolumn{2}{|c|}{$\begin{array}{c}\text { Harmonic } \\
\text { order }\end{array}$}} & \multicolumn{5}{|c|}{ Bus number } & \multirow{2}{*}{$\begin{array}{c}\text { AVG } \\
(\%)\end{array}$} \\
\hline & & 1 & 2 & 3 & 4 & 5 & \\
\hline \multirow{8}{*}{ 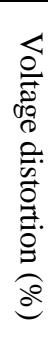 } & 5 & 0 & 0.044 & 1.386 & 3 & 2.96 & 1.478 \\
\hline & 7 & 0 & 0.045 & 1.386 & 3 & 2.84 & 1.454 \\
\hline & 11 & 0 & 0.003 & 0.228 & 1.924 & 0.95 & 0.621 \\
\hline & 13 & 0 & 0.001 & 0.085 & 0.695 & 0.58 & 0.272 \\
\hline & 17 & 0 & 0 & 0.015 & 0.416 & 0.24 & 0.134 \\
\hline & 19 & 0 & 0 & 0.0116 & 0.323 & 0.16 & 0.099 \\
\hline & 23 & 0 & 0 & 0.0077 & 0.111 & 0.06 & 0.036 \\
\hline & 25 & 0 & 0 & 0.0065 & 0.017 & 0.00 & 0.005 \\
\hline \multicolumn{2}{|c|}{ THDv (\%) } & 0 & 0.06 & 1.975 & 4.74 & 4.26 & 2.207 \\
\hline
\end{tabular}

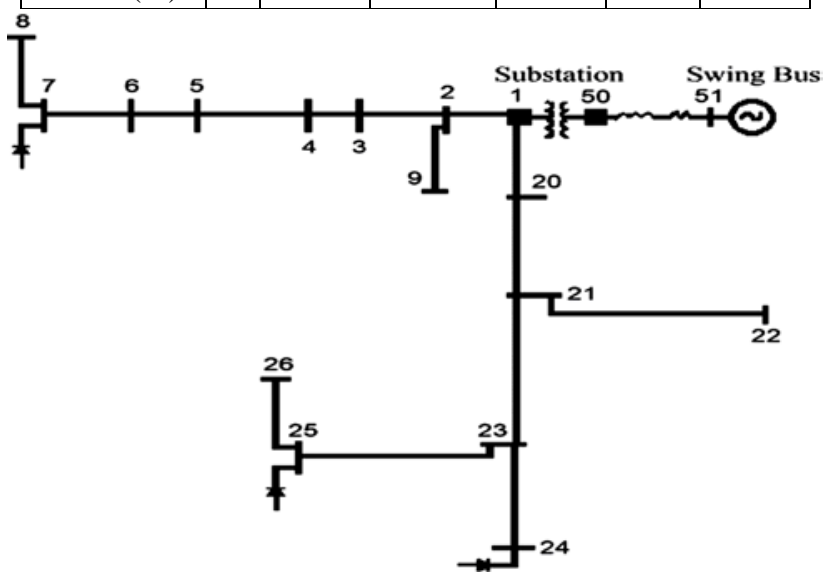

Fig. 8. 18-bus test system [4]-[7], [12].

In this case, sizes of the APFs are considered continuous. As expected, Table V and Fig.7 show that with increasing of harmonics order, injection harmonic current of D APF at bus 4 is reduced.

Table VI illustrates that the proposed method has been driven as desired and THDv on buses 4 and 5 is set to $4.74 \%$ and $4.26 \%$, respectively. Individual harmonic distortions have not exceeded the permissible value too. The maximum value of THDv and individual harmonic distortions after applying the proposed method are shown as bold in Table VI.

\section{B. 18-bus test system}

To assess the applicability of the proposed approach, the 18-bus test system is considered, (see Fig. 8). The system contains several linear loads and three 2.5 MW nonlinear loads at buses 7, 24 and 25, similar to the nonlinear load is used in the 5-bus test system. This network has been used in several published research papers with minor modifications, including displacement of some nonlinear loads [4]-[7], [12]. In this paper, this network is employed to provide an equal basis for comparison. 
TABLE VII

Initial Voltage Distortions For The 18-Bus System

\begin{tabular}{|l|l|l|l|l|l|l|l|l|l|}
\hline \multirow{2}{*}{$\begin{array}{c}\text { Bus } \\
\text { number }\end{array}$} & \multirow{2}{*}{$\begin{array}{c}\text { THDv } \\
(\%)\end{array}$} & \multicolumn{9}{|c|}{ Voltage distortion for each harmonic (\%) } \\
\cline { 3 - 11 } & & 5 & 7 & 11 & 13 & 17 & 19 & 23 & 25 \\
\hline 1 & 5.72 & 4.76 & 1.17 & 1.26 & 2.29 & 0.30 & 1.22 & 0.38 & 0.40 \\
\hline 2 & 6.18 & 5.24 & 0.80 & 1.17 & 1.95 & 0.58 & 2.01 & 0.55 & 0.54 \\
\hline 3 & 6.21 & 5.72 & 0.33 & 0.85 & 0.96 & 0.75 & 1.85 & 0.28 & 0.17 \\
\hline 4 & 6.20 & 5.91 & 0.36 & 0.67 & 0.54 & 0.73 & 1.42 & 0.10 & 0.13 \\
\hline 5 & 6.77 & 6.25 & 1.16 & 0.71 & 1.79 & 0.41 & 0.77 & 0.64 & 0.75 \\
\hline 6 & 6.74 & 6.15 & 1.38 & 0.80 & 1.96 & 0.16 & 1.01 & 0.36 & 0.27 \\
\hline 7 & 7.15 & 5.87 & 1.97 & 1.24 & 2.49 & 0.61 & 1.88 & 0.40 & 1.00 \\
\hline 8 & 7.16 & 5.88 & 1.97 & 1.24 & 2.49 & 0.61 & 1.88 & 0.40 & 1.00 \\
\hline 9 & 6.19 & 5.25 & 0.80 & 1.17 & 1.95 & 0.58 & 2.01 & 0.55 & 0.54 \\
\hline 20 & 8.74 & 6.16 & 3.07 & 2.34 & 4.71 & 0.91 & 0.71 & 0.18 & 0.19 \\
\hline 21 & 10.42 & 7.08 & 4.32 & 2.73 & 5.26 & 1.25 & 1.73 & 0.26 & 0.21 \\
\hline 22 & 10.43 & 7.08 & 4.33 & 2.73 & 5.26 & 1.26 & 1.73 & 0.26 & 0.21 \\
\hline 23 & 9.70 & 7.90 & 5.34 & 1.36 & 0.83 & 0.67 & 0.32 & 0.33 & 0.28 \\
\hline 24 & 10.75 & 8.51 & 6.10 & 1.16 & 1.95 & 0.51 & 0.66 & 0.34 & 0.28 \\
\hline 25 & 10.24 & 8.07 & 5.32 & 0.38 & 1.29 & 2.33 & 1.80 & 0.76 & 0.60 \\
\hline 26 & 10.24 & 8.08 & 5.32 & 0.38 & 1.29 & 2.33 & 1.80 & 0.76 & 0.60 \\
\hline AVG & $\mathbf{8 . 0 5}$ & 6.50 & 2.73 & 1.26 & 2.31 & 0.87 & 1.42 & 0.41 & 0.45 \\
\hline
\end{tabular}

TABLE VIII

Voltage Conditions After Employing The Proposed Method For The 18-Bus System

\begin{tabular}{|l|l|l|l|l|l|l|l|l|l|}
\hline \multirow{2}{*}{$\begin{array}{c}\text { Bus } \\
\text { number }\end{array}$} & \multirow{2}{*}{$\begin{array}{c}\text { THDv } \\
(\%)\end{array}$} & \multicolumn{9}{|c|}{ Voltage distortion for each harmonic (\%) } \\
\cline { 3 - 11 } & & 5 & 7 & 11 & 13 & 17 & 19 & 23 & 25 \\
\hline 1 & 2.91 & 1.92 & 1.44 & 0.81 & 1.14 & 0.44 & 0.34 & 0.47 & 0.45 \\
\hline 2 & 3.13 & 2.12 & 1.42 & 0.85 & 1.07 & 0.68 & 0.46 & 0.62 & 0.62 \\
\hline 3 & 2.94 & 2.18 & 1.28 & 0.74 & 0.78 & 0.84 & 0.44 & 0.34 & 0.24 \\
\hline 4 & 2.89 & 2.27 & 1.25 & 0.66 & 0.64 & 0.76 & 0.41 & 0.15 & 0.22 \\
\hline 5 & 2.98 & 2.29 & 0.95 & 0.54 & 0.94 & 0.45 & 0.40 & 0.68 & 0.85 \\
\hline 6 & 2.72 & 2.19 & 0.89 & 0.55 & 0.98 & 0.25 & 0.38 & 0.42 & 0.38 \\
\hline 7 & 3.09 & 2.10 & 0.94 & 0.77 & 1.24 & 0.72 & 0.49 & 0.44 & $\mathbf{1 . 0 7}$ \\
\hline 8 & 3.72 & 2.86 & 1.14 & 0.64 & 1.25 & 0.67 & 0.74 & 0.48 & $\mathbf{1 . 0 7}$ \\
\hline 9 & 3.21 & 2.10 & 1.48 & 0.87 & 1.07 & 0.69 & 0.74 & 0.59 & 0.67 \\
\hline 20 & 4.41 & 2.43 & 2.44 & 1.35 & 2.17 & 0.84 & 0.52 & 0.22 & 0.27 \\
\hline 21 & 5.00 & 2.76 & 2.94 & 1.39 & $\mathbf{2 . 3 0}$ & 1.10 & 0.47 & 0.24 & 0.21 \\
\hline 22 & $\mathbf{5 . 0 0}$ & 2.76 & 2.94 & 1.39 & $\mathbf{2 . 3 0}$ & 1.10 & 0.47 & 0.24 & 0.21 \\
\hline 23 & 4.31 & 2.78 & 2.93 & 0.92 & 0.62 & 0.69 & 0.52 & 0.47 & 0.31 \\
\hline 24 & 4.92 & $\mathbf{3 . 0 0}$ & $\mathbf{3 . 0 0}$ & $\mathbf{1 . 6 5}$ & 1.64 & 0.49 & 0.49 & 0.38 & 0.37 \\
\hline 25 & 4.99 & 2.55 & 2.72 & 0.54 & 1.64 & 2.24 & $\mathbf{1 . 4 2}$ & $\mathbf{0 . 7 6}$ & 0.60 \\
\hline 26 & 4.99 & 2.55 & 2.72 & 0.54 & 1.64 & 2.25 & $\mathbf{1 . 4 2}$ & $\mathbf{0 . 7 6}$ & 0.60 \\
\hline AVG & 3.83 & 2.43 & 1.91 & 0.89 & 1.34 & 0.89 & 0.61 & 0.45 & 0.51 \\
\hline
\end{tabular}

Table VII illustrates the obtained simulation results in no APF state. As shown in Table VII, the resulting THDv at all buses is, on average, $8.05 \%$, which represents a rather unallowable harmonic distortion level in reference to the IEEE standard limit of $5 \%$. The distortion levels after employing proposed method are given in Table VIII. The maximum 
TABLE IX

Results For The Proposed Methods Applied To 18-Bus SYSTEM

\begin{tabular}{|l|l|l|l|l|}
\hline \multicolumn{1}{|c|}{$\begin{array}{l}\text { APFs } \\
\text { ON }\end{array}$} & $\begin{array}{c}\text { APF } \\
\text { Bus }\end{array}$ & $\begin{array}{c}\text { AVG. } \\
\text { THDv } \\
(\%)\end{array}$ & $\begin{array}{c}\text { Max.THDv } \\
(\%) \\
\text { (At bus) }\end{array}$ & $\begin{array}{c}\text { Total } \\
\text { Injected } \\
\text { current } \\
(\%)\end{array}$ \\
\hline None & - & 8.05 & $10.75(24)$ & - \\
\hline A & 24 & 6.16 & $8.75(25)$ & 5.0 \\
\hline A, B & 24,21 & 4.64 & $6.53(25)$ & 9.0 \\
\hline A, B, C & $24,21,8$ & 3.96 & $5.58(21)$ & 11.2 \\
\hline A, B, C, D & $24,21,8,25$ & 3.83 & $5(21,22)$ & 12.5 \\
\hline
\end{tabular}

values are within acceptable ranges that they are shown as bold in Table VIII. As shown in Table VIII, the resulting THDv at all buses is, on average, $3.83 \%$.

The installed APF sizes are considered as integer multiples of $0.1 \%$. The size of each APF is given in Table I. The maximum voltage harmonic distortions and THDv are considered to be 3 and 5\%, respectively, so that the results may be compared with [10] and [12].

All buses are considered as candidates for APF installation and up to the 25th harmonic are considered for compensation.

After applying the proposed method, A, B, C and D APFs with 5\%, 4\%, 2.2\% and 1.3\% RMS injection current are installed on buses 24, 21, 8 and 25,respectively. Therefore the total size of APFs is set to $12.5 \%$ and the number of APF to 4. See Table IX. Similar cases study in [10] and [12] are resulted in $12 \%$ and $13 \%$ total size and 4 and 5 APFs, respectively. The proposed method has reduced the number of APFs in comparison with [10] and total injected currents in comparison with [12], respectively.

As a result, it is expected that the maximum current to be injected by APF in bus 24, (see Table X).

GRG2 is a nonlinear optimizing program that uses generalized reduced gradient algorithm. Provided with a starting point, GRG2 can further improve the objective function, and if possible, find optimal solution. GRG2 stops searching for a better solution when the Kuhn-Tucker conditions are satisfied to within tolerance, or if the change of the objective function value is very small for a default number of iterations. GRG2 solution times are greatly improved by using a good starting point. GRG2 is used to improve the APF placement and sizing when higher accuracy is required [10].

The GA is a search algorithm based on natural genetic structure. Its theory is based on "a better chance of surviving for a population with a better fitness". The GA can be used for the estimation of global minimum or maximum values of a function even in the presence of some local extermum. In other words, it will not be trapped in the local extermum and searches for the global one. The procedure is to initially generate, namely, a population of chromosomes; representing
TABLE X

Comparative Study For The 18-Bus System

\begin{tabular}{|c|c|c|c|}
\hline & \multicolumn{3}{|c|}{ Methods } \\
\hline & $\begin{array}{c}\text { GRG2 } \\
{[10]}\end{array}$ & $\begin{array}{l}\text { GA } \\
{[12]}\end{array}$ & $\begin{array}{l}\text { Proposed } \\
\text { method }\end{array}$ \\
\hline $\begin{array}{l}\text { APF bus (RMS of injection } \\
\text { current \%) }\end{array}$ & $\begin{array}{l}7(1) \\
8(1) \\
21(1) \\
24(7) \\
25(2)\end{array}$ & $\begin{array}{c}- \\
8(3) \\
21(3) \\
24(5) \\
25(2)\end{array}$ & $\begin{array}{c}- \\
8(2.2) \\
21(4) \\
24(5) \\
25(1.3)\end{array}$ \\
\hline Total injected currents (\%) & 12 & 13 & 12.5 \\
\hline Number of APFs & 5 & 4 & 4 \\
\hline Avrage THDv (\%) & 2.65 & 3.62 & 3.83 \\
\hline Maximum THDv (\%) & 4.19 & 5 & 5 \\
\hline Max THDv on buses & 24 & 21,22 & 21,22 \\
\hline
\end{tabular}

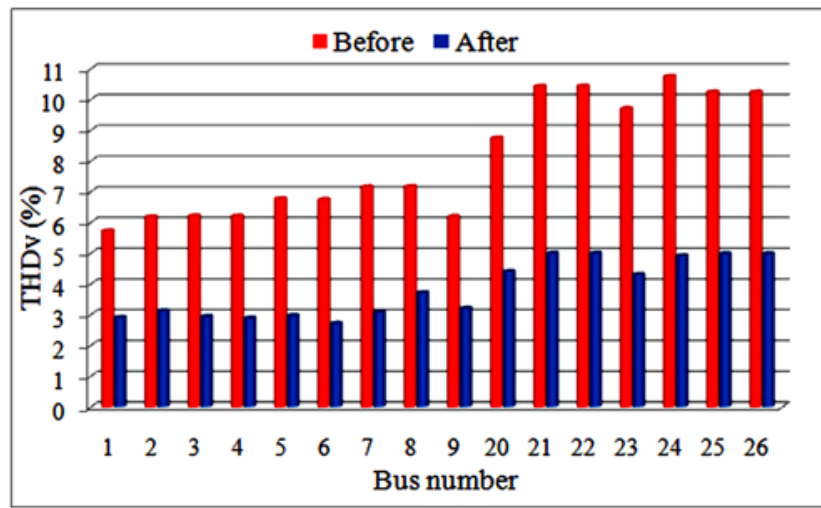

Fig. 9. Comparison between THDv before and after employing the proposed method for the 18-bus system.

decision-making variables. Using two operators, namely, crossover and mutation, new chromosomes are generated. In [12], a genetic based algorithm is employed for APF allocation and sizing.

The proposed method has reduced the number of APFs in comparison with [10] and total injected currents in comparison with [12], respectively. The results of comparison are shown in Table X.

All THDv values are within acceptable ranges. Comparison between THDv before and after APF installation is shown in Fig. 9.

A comparative study of the results in Table $\mathrm{X}$ reveals that the method proposed in the present paper can determine much better solutions.

\section{CONCLUSIONS}

This paper proposed fuzzy based new solution for allocation 
and sizing multiple APFs. We define new membership functions for identification of probable APF locations in distribution systems. The product operator shows better performance than the AND operator because all harmonics are considered in computing membership function. A new algorithm for selection of APF nodes is presented and MAPSO is employed for final sizing of the APFs at the nodes. Fuzzy based approaches involve less computational burden. The proposed algorithm has been successfully implemented to the 5-bus and the 18-bus test system. Comparison with the other heuristic based methods and an analytical optimization technique shows that the proposed method produces better solutions. The proposed method has reduced the number of APFs in comparison with [10] and total injected currents in comparison with [12], respectively. We consider the weighting factors for harmonic voltage membership $\left(w_{v}\right)$ and harmonic current membership ( $w_{i}$ ), 0.6 and 0.4 , respectively, because in our objective function, the distortions of voltage more important than the distortions of current. It is clear that the amount of the membership functions had a clear effect on the objective values. Therefore finding the best values for $w_{v}$ and $w_{i}$ is our great goal for providing the optimum amount for the objective function in the next research. Decision variables of the optimization algorithm will be defined as two weighting factors which are $w_{v}$ and $w_{i}$.

\section{ACKNOWLEDGMENT}

This research was supported by Semnan University. Also the authors gratefully acknowledge the support of the talented office of Semnan University for supporting the current research.

\section{REFERENCES}

[1] J. Arrillaga and N. R. Watson, Power System Harmonics, Second Edition, John Wiley \& Sons, 2003.

[2] B. Singh and K. Al-Haddad, "A review of active filters for power quality improvement,” IEEE Trans. Ind. Electron., Vol. 46, No. 5, pp. 960-971, Oct. 1999.

[3] W. M. Grady, M. J. Samotyj, and A. H. Noyola, "Survey of active power line conditioning methodologies," IEEE Trans. Power Del., Vol. 5, No. 3, pp. 1536-1542, Jul. 1990.

[4] W. M. Grady, M. J. Samotyj, and A. H. Noyola, "Minimizing network harmonic voltage distortion with an active power line conditioner," IEEE Trans. Power Del., Vol. 6, No. 4, pp. 1690-1697, Oct. 1991.

[5] W. K. Chang, W. M. Grady, and M. J. Samotyj, "Meeting IEEE-519 harmonic voltage and voltage distortion constraints with an active power line conditioner," IEEE Trans. Power Del., Vol. 9 No. 3, pp. 1531-1537, Jul. 1994.

[6] W. M. Grady, M. J. Samotyj, and A. H. Noyola, "The application of network objective functions for actively minimizing the impact of voltage harmonics in power systems," IEEE Trans. Power Del., Vol. 7 No. 3, pp.
1379-1386, Jul. 1992.

[7] W. K. Chang, W. M. Grady, and M. J. Samotyj, “A practical method for sitting and sizing multiple active power line conditioners in a power system," in proceeding of IEEE Transmission and Distribution, Chicago, 1994.

[8] W. K. Chang, W. M. Grady, and P. Verde, "Determining the optimal current injection and placement of an active power line conditioner for several harmonic-related network correction strategies," the Sixth International Conference on Harmonics in Power Systems, Bologna, Italy, 1994.

[9] W. K. Chang, W. M. Grady, and M. J. Samotyj, "Controlling harmonic voltage and voltage distortion in a power system with multiple active power line conditioners," IEEE Trans. Power Del., Vol. 10, No. 3, pp. 1670-1676, Jul. 1995.

[10] W. K. Chang and W. M. Grady, "Minimizing harmonic voltage distortion with multiple current constrained active power line conditioners,” IEEE Trans. Power Del., Vol. 12, No. 2, pp. 837-843, Apr. 1997.

[11] Y. Y. Hong and Y. K. Chang, "Determination of locations and sizes for active power line conditioners to reduce harmonics in power systems," IEEE Trans. Power Del., Vol. 11, No. 3, pp. 1610-1617, Jul. 1996.

[12] R. Keypour, H. Seifi, and A. Yazdian-Varjani, "Genetic based algorithm for active power filter allocation and sizing," Elsevier Electric Power Systems Research, Vol. 71, No. 1, p.p. 41-49 Sep. 2004.

[13] I. Ziari and A. Jalilian, "A new approach for allocation and sizing of multiple active power-line conditioners," IEEE Trans. Power Del., Vol. 25, No. 2, pp. 1026-1035 Apr. 2010.

[14] I. Ziari and A. Jalilian, "Optimal harmonic power flow using an ant colony system-based algorithm," in Proceeding of Universities Power Engineering Conference, pp. 1-4, Padova, Sep. 2008.

[15] S. Mishra and C. N. Bhende, "Bacterial foraging technique-based optimized active power filter for load compensation,” IEEE Trans. Power Del., Vol. 22, No. 1, pp. 457-465, Jan. 2007.

[16] W. Yand-song, S. Hua, L. Xue-min, L. Jun, G. Song-bo, "Optimal allocation of the active filters based on the tabu algorithm in distribution network," in Proceeding of Electrical and Control Engineering, Wuhan, China, Jun. 2010.

[17] H. Chin and W. Lin, "Capacitor placement for distribution systems with fuzzy algorithm," in proceeding of IEEE Region 10's Ninth Annual International Conference, Theme: Frontiers of Computer Technology, Vol. 2, pp. 1025-1029, Aug. 1994.

[18] H. C. Chin, "Optimal shunt capacitor allocation by fuzzy dynamic programming," Elsevier Electric Power Systems Research, Vol. 35, No. 2, pp. 133-139, Nov. 1995.

[19] H. N. Ng and M. M. A. Salama, "Fuzzy optimal capacitor sizing and placement," in Proceeding of Canadian Conference on Electrical and Computer Engineering, Vol. 2, pp. 680-683, Calgary, Alta., Canada, Sep. 1995.

[20] H. N. Ng, M. M. A. Salama, and A. Y. Chikhani, "Capacitor placement in distribution systems using fuzzy technique," in Proceeding of Canadian conference on Electrical and Computer Engineering, Vol. 2, pp. 790-793, Calgary, Alta., Canada, May, 1996.

[21] C. Su and C. Tsai, "A new fuzzy-reasoning approach to optimum capacitor allocation for primary distribution systems," in Proceeding of IEEE International Conference 
Industrial Technology, pp. 237-241, Shanghai, China, Dec. 1996.

[22] C. T. Su, G. R. Lii, and C. C. Tsai, "Optimal capacitor allocation using fuzzy reasoning and genetic algorithms for distribution systems," Elsevier Mathematical and Computer Modelling, Vol. 33, No. 6-7, pp. 745-757, Apr. 2001.

[23] S. F. Mekhamer, S. A. Soliman, M. A. Moustafa, and M. E. El-Hawary, "Application of fuzzy logic for reactive power compensation of radial distribution feeders," IEEE Trans. Power Syst., Vol. 18, No. 1, pp.206-213, Feb. 2003.

[24] M. A. S. Masoum, A. Jafarian, M. Ladjevari, E. F. Fuchs, and W. M. Grady, "Fuzzy approach for optimal placement and sizing of capacitor banks in the presence of harmonics," IEEE Trans. Power Del., Vol. 20, No. 2, pp. 822-829, Apr. 2004.

[25] S. K. Bhattacharya and S. K. Goswami, “A new fuzzy based solution of the capacitor placement problem in radial distribution system," Elsevier Expert Systems with Applications, Vol. 36, No. 3, pp. 4207-4212, Apr. 2009.

[26] B. Gasbaoui, A. Chaker, A. Laoufi, A. Abderrahmani, and B. Allaoua, "Optimal placement and sizing of capacitor banks using fuzzy-ant approach in electrical distribution systems," "Leonardo Electronic Journal of Practices and Technologies, Vol. 9 No. 16, pp. 75-88, Jun. 2010.

[27] S. M. Tabatabaei and B. Vahidi, "Bacterial foraging solution based fuzzy logic decision for optimal capacitor allocation in radial distribution system," Elsevier Electric Power Systems Research, Vol. 81, No. 4, pp. 1045-1050 Apr. 2011.

[28] M. E. El-Hawary, "Electric Power Applications of Fuzzy Systems.” Piscataway, NJ: IEEE Press, 1998.

[29] J. Kennedy, R. Eberhart, "Particle swarm optimization," IEEE International Conference on Neural Networks, Vol. 4, pp. 1942-1948, Perth, WA, Australia, Nov. 1995.

[30] N. Amjady and H. R. Soleymanpour, "Daily hydrothermal generation scheduling by a new modified adaptive particle swarm optimization technique," Elsevier Electric Power Systems Research, Vol. 80 No. 6, p.p. 723-732, Jun. 2010.

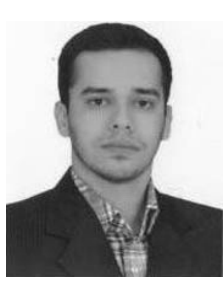

Amir Moradifar was born in Tehran, Iran, on September 21, 1986. He has received the B.Sc. degree in electrical engineering from Semnan University, Semnan, Iran in 2008 and the M.Sc. degree in electrical engineering from Semnan University, Semnan, Iran in 2010. His research interests include electric power quality, power system harmonics, optimization techniques applied to electrical systems and distribution system planning.

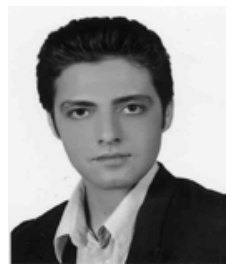

Hassan Rezai Soleymanpour was born in Karaj, Iran, on April 5, 1985. He has received the B.Sc. degree in electrical engineering from Hormozgan University, Hormozgan, Iran in 2007 and the M.Sc. degree in electrical engineering from Semnan University, Semnan, Iran in 2010. His research interests include power system operation and planning and optimization techniques applied to electrical systems. 